\title{
Téoros
}

Revue de recherche en tourisme

\section{L'évolution des flux de touristes dans les grandes régions du monde}

\section{Illusions de perspectives en tourisme international}

\section{Paul Bodson et Jean Stafford}

Volume 6, numéro 3, décembre 1987

Le tourisme, phénomène mondial

URI : https://id.erudit.org/iderudit/1080598ar

DOI : https://doi.org/10.7202/1080598ar

Aller au sommaire du numéro

Éditeur(s)

Université du Québec à Montréal

ISSN

0712-8657 (imprimé)

1923-2705 (numérique)

Découvrir la revue

Citer cet article

Bodson, P. \& Stafford, J. (1987). L'évolution des flux de touristes dans les

grandes régions du monde : illusions de perspectives en tourisme

international. Téoros, 6(3), 2-6. https://doi.org/10.7202/1080598ar 
Illusions de perspectives en tourisme international
L'évolution des flux de touristes dans les grandes régions du monde
Depuis 1945, les flux touristiques internationaux ont connu un développement extraordinaire. Cet accroissement est le fruit, à la fois, de découvertes technologiques et des "trente glorieuses" annees d'enrichissement collectif résultant d'une expansion économique sans exemple dans l'histoire modeme (')

Activité jadis réservée à des groupes privilégiés, le tourisme est progressivement devenu accessible à d'autres couches de population. Cette rupture d'avec le "tourisme classique" a entraîné d'importants bouleversements $^{(2)}$. Le tourisme de masse a provoqué un changement complet de conception et de pratique dans le domaine. Lentement, par la force et l'ampleur même du phénnomène, le tourisme international est devenu une veritable industrie. Les ideaux qui prétendaient l'animer dans les années 1950 (ouverture aux autres sociétés, communication) se sont progressivement estompés et sont devenus de vagues principes dont

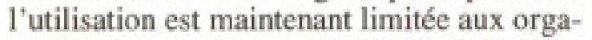
nismes internationaux (O.N.U. O.M.T. ).

Le tourisme est aujourd 'hui tributaire d'une organisation qui laisse peu de place aux bons sentiments. Le transport aérien est un exemple caractéristique de cente évolution. Les enjeux économiques y sont de plus en plus régis par la loi implacable de l'offre et de la demande. Parallèlement, les produits touristiques évoluent vers une plus grande standardisation. La pression de la concurrence exige aussi une planification serrée et spécifique. Le tourisme international est ainsi devenu une industrie semblable aux autres industries, prenant place à leur côté dans l'ensemble de l'économie mondiale. C'est dans cette perspective que nous avons étudié l'évolution des flux touristiques (captes par les arrivées de touristes) au niveau du monde et au niveau de ses principales régions.

"Economistes, professeurs au Dejpartement d'études urbaines et touristiques, UOAM

\section{Croissance et stabilité du tourisme international}

Le tourisme international a connu une croissance rapide de 1950 à 1960 , ainsi qu'en témoigne le tableau no 1. (page 3).

La progression s'est légèrement ralentie entre 1960 et 1970 pour diminuer à nouveau dans les années 1970-1980 avec un TAAM de 5.7. La periode 1980-1985 est marquée par une nouvelle baisse de l'accroissement par rapport aux années antérieures puisqu'on se retrouve avec un TAAM de $3.6 \%$.

Cette glissade graduée du taux de croissance annuel moyen a une base technique. Les points de référence des taux de croissance concernent des masses de touristes de plus en plus importantes; un plus grand nombre d'unités (arrivées de touristes) est ainsi requis pour assurer la stabilité des taux de croissance. Il existe aussi une raison fconomique à la diminution des taux de croissance. La crise pétrolière de 1973 a mis fin à la période d'enrichissement collectif systématique. Il ne fait guère de doute que la stagnation des revenus réels dans de nombreux pays du monde a freine la croissance des flux internationaux de touristes $^{(3)}$.

La dure récession de la période 1981-1982 a, elle aussi, exercé un impact négatif sur l'évolution du tourisme international et tout particulièrement aux États-Unis et au Canada ${ }^{(4)}$. Très heureusement, la baisse généralisće de l'ensemble des tarifs aériens, du moins pour les destinations touristiques, a joué un rồle stabilisateur ${ }^{(5)}$, en compensant partiellement les pertes dues à la baisse du pouvoir d'achat des touristes.

Dans l'ensemble, les prévisions concernant le tourisme international ont été infirmées. L'O.M.T. prévoyait les résultats suivants pour la période 1981-1985 ${ }^{6)}$. (voir tableau 2, page 3 ).

Ces données confirment combien le tourisme fait désormais partie intégrante de l'économie mondiale et est soumis aux aléas de son évolution.

La banalisation du tourisme et son intégration progressive à l'économie a eu pour principale conséquence d'exacerber les luttes pour les marches touristiques et d'orienter l'économie touristique en fonction des avantages comparatifs internationaux. Il en est résulté des évolutions inégales dans 1"industrie du tourisme, au niveau des différentes régions du monde.

\section{Les marchés touristiques internationaux depuis 1965}

L'évolution des marchés touristiques internationaux de 1965 a 1985 manifeste des changements majeurs dans la répartition spatiale des touristes.

Les grandes régions polarisatrices de touristes (Amérique du Nord, Europe) ont vu se réduire lentement mais systématiquement leurs parts respectives du marché touristique. L'Amérique du Nord apparaît la région du monde la plus perdante. En vingt ans, sa part du marché mondial est passée de $17,2 \%$ en 1965 a $9,6 \%$ en 1985 . L'Europe, à son tour, a vu son attrait proportionnellement se réduire puisque sa part du marché est passée de $74,2 \%$ en 1965 a $66,8 \%$ en 1985 . Par contre, l'Asie de l'Est et le Pacifique ont connu un développement foudroyant avec un taux d'accroissement annuel moyen de $17 \%$ entre 1965 et 1985. (voir tableau 3, page 3).

Viennent ensuite l'Afrique (TAAM $=9,7$ ), l'Asie du Sud (TAAM $=9,7)$ puis l'Amérique du Sud (TAAM $=8,6$ ), le MoyenOrient (TAAM $=5,7$ ). Seules l'Europe et l'Amérique du Nord ne dépassent pas le TAAM de $5 \%$. On soulignera par contre que les taux se réfêrent à des populations de touristes proportionnellement plus importantes pour ces deux régions.

De plus, il ne faut pas perdre de vue que les principales zones émettrices et réceptrices de touristes se retrouvent dans les pays oceidentaux. C'est une idée reçue de croire que les pays riches ne constituent que le pôle de la demande touristique internationale; en fait, ces pays de façon inégale, attirent la majeure partie de la clientèle mondiale et done des recettes issues du tourisme.

\section{L'évolution récente (1981-1985)}

L'évolution récente du partage des marchés touristiques internationaux suit à peu près les mêmes lignes de forces que la longue perriode 1965-1985.

Comme on peut le constater dans le tableau no 4, l'importance de la crise économique de 1981-1982 se répercute dans la quasitotalité des grandes régions du monde. Seule l'Asie de l'Est maintient le niveau de son indice supérieur à 100 sur toute la période 1981-1985. La croissance des flux touristiques reprend néanmoins progressivement dans l'ensemble des régions, En 1985, seule l'Amérique du Nord se situe encore endessous du niveau de 1981 . 
Tableau no 1

Taux d'accroissement annuel moyen (TAAM) du tourisme international de 1950 à 1985

\begin{tabular}{lcccc}
\hline & $1950 / 1960$ & $1960 / 1970$ & $1970 / 1980$ & $1980 / 1985$ \\
\hline TAAM & 10.6 & 8.7 & 5.7 & 3.6
\end{tabular}

Source: Chiffres calculés à partir des données de l'OMT (arrivées internationales de touristes).
Tableau no 2

Prévisions d'arrivées internationales 1981-1985

\begin{tabular}{lllc}
\hline Années & $\begin{array}{l}\text { Arrivées } \\
\text { (en millions) }\end{array}$ & \multicolumn{2}{l}{$\begin{array}{l}\text { Prévisions } \\
\text { Hypothèses (en millions) }\end{array}$} \\
& & Faible & Forte \\
\hline 1981 & 290.0 & 294.3 & 320.3 \\
1982 & 286.9 & 307.1 & 344.1 \\
1983 & 293.9 & 320.0 & 370.0 \\
1984 & 315.3 & 333.0 & 398.0 \\
1985 & 332.9 & 345.9 & 428.4 \\
\hline
\end{tabular}

Source: O.M.T., 1981.

Tableau no 3

Part du marché mondial (en \%) selon les régions et les périodes

\begin{tabular}{lllllllll}
\hline Années & $\begin{array}{l}\text { Amérique } \\
\text { du Nord }\end{array}$ & $\begin{array}{l}\text { Amérique } \\
\text { du Sud }\end{array}$ & Europe & $\begin{array}{l}\text { Moyen- } \\
\text { Orient }\end{array}$ & Afrique & $\begin{array}{l}\text { Asie de } \\
\text { l'est }\end{array}$ & $\begin{array}{l}\text { Asie } \\
\text { du sud }\end{array}$ & Total \\
\hline 1965 & 17,2 & 3,4 & 74,2 & 2,1 & 1,3 & 1,4 & 0,4 & 100 \\
1970 & 17,1 & 5,8 & 70,7 & 1,2 & 1,5 & 3,0 & 0,7 & 100 \\
1975 & 13,6 & 6,3 & 72,8 & 1,4 & 2,2 & 4,0 & 0,7 & 100 \\
1980 & 12,5 & 7,2 & 69,9 & 2,0 & 2,1 & 5,5 & 0,8 & 100 \\
1985 & 9,6 & 6,0 & 66,8 & 2,1 & 3,7 & 10,9 & 0,9 & 100 \\
TAAM & 2,6 & 8,6 & 5,0 & 5,7 & 9,7 & 17,0 & 9,7 & \\
en \% & & & & & & & &
\end{tabular}

Tableau no 4

Évolution indicielle des marchés touristiques internationaux entre 1981 et 1985

(base 100 en 1981)

\begin{tabular}{lllllllll}
\hline Années & $\begin{array}{l}\text { Amérique } \\
\text { du Nord }\end{array}$ & $\begin{array}{l}\text { Amérique } \\
\text { latine }\end{array}$ & Europe & $\begin{array}{l}\text { Moyen- } \\
\text { Orient }\end{array}$ & Afrique & $\begin{array}{l}\text { Asie de } \\
\text { l'est }\end{array}$ & $\begin{array}{l}\text { Asie } \\
\text { du sud }\end{array}$ & Monde \\
\hline 1981 & 100 & 100 & 100 & 100 & 100 & 100 & 100 & 100 \\
1982 & 95 & 95 & 99 & 113 & 95 & 104 & 99 & 99 \\
1983 & 91 & 104 & 102 & 98 & 98 & 114 & 102 & 102 \\
1984 & 90 & 112 & 110 & 106 & 107 & 133 & 101 & 109 \\
1985 & 90 & 115 & 115 & 115 & 113 & 157 & 104 & 115 \\
\hline
\end{tabular}

Tableau no 5

Prévisions du nombre d'arrivées internationales Niveau mondial

\begin{tabular}{lll}
\hline Année & Prévisions & $\begin{array}{l}\text { Marge statistique } \\
\text { de fluctuation }\end{array}$ \\
\hline 1986 & 341.18 & \pm 36.23 \\
1987 & 343.45 & \pm 40.78 \\
1988 & 365.60 & \pm 46.32 \\
1989 & 377.54 & \pm 52.96 \\
1990 & 389.80 & \pm 60.02 \\
1995 & 441.89 & \pm 118.58 \\
\hline
\end{tabular}

Tableau no 6

Répartition spatiale du marché mondial du tourisme (pourcentage régional du marché)

Prévisions

\begin{tabular}{lrrrr}
\hline Régions & $\begin{array}{r}\text { Années } \\
\mathbf{1 9 8 6}\end{array}$ & $\mathbf{1 9 9 0}$ & $\mathbf{1 9 9 5}$ & $\mathbf{2 0 0 0}$ \\
\hline Amérique du Nord & 9,82 & 8,37 & 6,83 & 5,46 \\
Amérique Latine & 6,22 & 6,40 & 6,69 & 7,09 \\
Europe & 67,95 & 68,40 & 69,28 & $\mathbf{7 0 , 5 4}$ \\
Moyen-Orient & 2,25 & 2,44 & 2,70 & 3,05 \\
Afrique & 2,69 & 2,57 & 2,48 & 2,50 \\
Asie de l'Est-Pacifique & 10,37 & 11,32 & 11,75 & 11,24 \\
Asie du Sud &, 70 &, 5 &, 27 &, 12 \\
\hline TOTAL: & 100 & 100 & 100 & $\mathbf{1 0 0}$ \\
\hline
\end{tabular}


L'Asie de l'Est est la région où l'augmentation des arrivées de touristes devance de loin toutes les autres régions. L'Amérique du Nord, à l'opposé, est la région la plus affectée par la réduction des arrivées touristiques: son indice descend progressivement jusqu'à 90. L'Asie du Sud connaît aussi un développement ralenti entre 1983 et 1985 . Hormis ce cas, la répartition des marchés touristiques entre les régions étudiées, semble inscrite dans la tendance longue qui s'annonce dès les atinées 1970 .

\section{L'avenir}

L'essor des moyens de transports modernes, en particulier à moyenne et longue distance, a rendu massivement accessibles des localisations peu fréquentées ou réservées à des clientèles privilégiées. L'industrie du tourisme a tiré profit de cette situation en développant une gamme diversifiée de destinations nouvelles ou exploitées sur une échelle limitée et en les rendant disponibles à des classes de revenus de plus en plus étendues. Ce contexte, associé à la hausse du revenu réel durant les trente dernières années pour une partie de la population mondiale a abouti à un accroissement insoupçonné des flux touristiques et a une modification de leur répartition entre les grandes régions du monde. Le phénomène continue à évoluer. Nous tenterons maintenant sur la base de l'analyse des données disponibles de discerner les lignes directrices de l'évolution future.

\section{Niveau mondial}

Au niveau mondial, les flux touristiques (captés par les arrivées internationales) suivent une tendance linéaire systématique $\left(\mathrm{R}^{2}\right.$ $=.95$ ). Du point de vue prévisionnel, les chiffres mondiaux ont cependant été calculés sur la base des prévisions au niveau des régions du monde de manière à assurer l'harmonisation des résultats.

Le taux de croissance annuelle (basé sur les prévisions par cible) dépasserait les $3 \%$ audelà de 1990 pour descendre progressivement à $2,67 \%$ en 1995 et 1,87 en 2000 . Ces résultats sont, toutefois, marqués d'une marge statistique de fluctuation. Compte tenu de cette marge, les prévisions d'artivées internationales pour l'ensemble du monde se présentent comme suit. (voir tableau 5, page 3).

Même en tenant compte de la marge statistique d'imprécision, les prévisions suggèrent que l'industrie du tourisme est destinée à de nouvelles mutations en raison de la masse de clients dont elle devra tenir compte.

\section{Les régions du monde}

La crise économique survenue en 1981-1982 a profondément perturbé l'évolution des flux touristiques dans la majorité des pays du monde et contribué à modifier les répartitions spatiales de clientèles. Dans l'ensemble, les années de crise créent une rupture, suivie d'une reprise (sauf pour l'Amérique du Nord), mais les lignes de force de l'évolu- tion ultérieure restent encore imprécises faute de données, grevant d'incertitude à la fois le choix des relations-synthese et les projections qui en résultent. En dépit de ces restrictions, les séries disponibles de l'O.M.T. manifestent de puissantes régularités temporelles qui permettent d'élaborer des hypothèses de projections relativement solides.

\section{L'Amérique du Nord (États-Unis, Canada)}

Parmi les grandes régions du monde, 1'Amérique du Nord (États-Unis, Canada) apparaît particulièrement touchée par un ralentissement du tourisme international. Le cours du dollar n'est pas étranger à cette situation ${ }^{(7)}$. Une courbe parabolique capte avec une assez grande précision la régularité de la série. Il en résulte une image de l'avenir relativement pessimiste (TAAM $=-, 52 \%$ entre 1985 et 1990 et $\mathrm{TAAM}=-1,28 \%$ entre $1990 \mathrm{et}$ 1995).

Cependant, les fluctuations récentes du dollar et les modifications internationales de pouvoir d'achat qui leur sont liées pourraient rapidement obliger à une révision des prévisions.

\section{L'Amérique latine}

L'Amérique latine a connu une baisse de clientèle dès 1980. Pạr contre, un redressement s'amorce à partir de 1983. La crise semble avoir perturbé l'allure de la regularité temporelle en créant une rupture à la baisse, à partir de laquelle se redéveloppe l'evolution en croissance. Si l'on tient compte de cette rupture, $\mathrm{I}^{+} \mathrm{Amé}$ rique latine s'octroit progressivement une part de plus en plus grande de la clientèle touristique mondiale grâce à un taux de croissance annuel supérieur à $3,87 \%$ jusqu'en 1990 et supérieur à 3,24 jusqu'en 1995 .

\section{L'Europe}

En dépit du ralentissement de croissance issu de la crise de 1981-1982, l'Europe garde son attrait traditionnel. Son rythme de croissance annuel se maintiendra supérieur à $3,28 \%$ jusqu'en 1990 et supérieur a $2,55 \%$ jusqu'en 1995. Comparativement à l'Asie de l'Est, au Moyen-Orient, à l'Amérique Latine, ces taux peuvent paraitre faibles. En fait, ils correspondent à des volumes importants de clientèle, dont le poids est déterminant dans l'évolution de la répartition mondiale du marché touristique.

\section{Le Moyen-Orient}

Le Moyen-Orient présente la particularité d'avoir subi le choc d'une baisse de clientèle en 1983 plutôt qu'en 1982 . Le contextè politique explique sans doute cette particularité. Le redressement de la situation s'effectue rapidement. En dépit du climat d'insécurité issu de conflits politiques et sociaux persistants, cette région du monde semble destinée à une forte croissance de clientèle (plus de 5,29\% jusqu'en 1990 et plus de 4,50 jusqu'en 1995).

\section{Afrique}

Malgré une croissance qui lui a assuré une part de plus en plus grande dans le tourisme international $(1,3 \%$ en 1965 et $3,7 \%$ en 1985), l'Afrique comporte un profil temporel qui semble la destiner à un taux de croissance ralenti et relativement bas (baisse de $4,0 \%$ en 1986 à $2,18 \%$ en 1990 et à $1,97 \%$ en 1995). Si ce profil se confirme, il en résultera une stabilisation progressive du rôle de l'Afrique au sein du tourisme mondial (environ $2,5 \%$ ).

\section{L'Asie de I'Est et le Pacifique}

Depuis 1975 , le rythme de croissance des "arrivées internationales" dans l'Asie de l'Est et le Pacifique a été tel qu'il a créé un glissement massif du marché du tourisme mondial vers cette région. Tout en restant elevé, les taux de croissance dans cette région sont néanmoins voués à un rạlentissement (baisse de 7,07\% en 1986 à $4,86 \%$ en 1990 et 2,30 en 1990). Par contre, ces taux de croissance permettront de consolider le rôle croissant de cette région dans le tourisme international $(10,37 \%$ du volume mondial des arrivées internationales en $1986,11,32 \%$ en 1990 et $11,75 \%$ en 1995 ).

\section{Asie du Sud}

Assez étonnament, l'Asie du Sud ne semble pas entraînée dans le mouvement de croissance du tourisme international qui anime l'Asie de l'Est et le Pacifique. La zone concerne une clientèle relativement limitée $(.9 \%$ du marché mondial en 1985). Elle semble avoir très légèrement subi l'effet de la crise de 1981-1982. Par contre, la régularité sousjacente à lạ série porte un ferment de ralentissement. Projetée sur l'avenir, cette caractéristique génère une réduction progressive de la clientèle qui aboutit à pratiquement exclure cette zone de l'essor contemporain du tourisme.

\section{Conclusion}

Les évolutions et les perspectives d'évolution que nous avons évoquées ont créé et créeront de profonds changements dans l'industrie du tourisme mondial. Le volume de la clientèle touristique semble destiné à croître régulièrement durant les prochaines années dans le prolongement des tendances lourdes discernées dans le passé.

Les enjeux économiques sous-jacents à cette évolution sont énormes. Par contre, les régions du monde en seront affectées de façon inćgale. (voir tableau 6, page 3).

Dans cette nouvelle répartition, l'Amérique du Nord apparaît particulièrement désavantagée (elle perd pratiquement $3 \%$ du marché mondial du tourisme entre 1986 et 1995). Les autres perdants sont l'Asie du Sud (perte de $.43 \%$ du marché entre 1986 et 1995 ) et l'Afrique (perte de ,21 du marché). Les bénéficiaires sont, dans l'ordre, l'Asie de l'Est $(1,38 \%$ entre 1986 et 1995$)$, l'Europe $(1,33 \%$ entre 1986 et 1995$)$, l'Amérique Latine $(47 \%)$ et le Moyen-Orient $(.45 \%)$. 
Graphique 1: Prévisions des arrivées des touristes en Amérique du nord

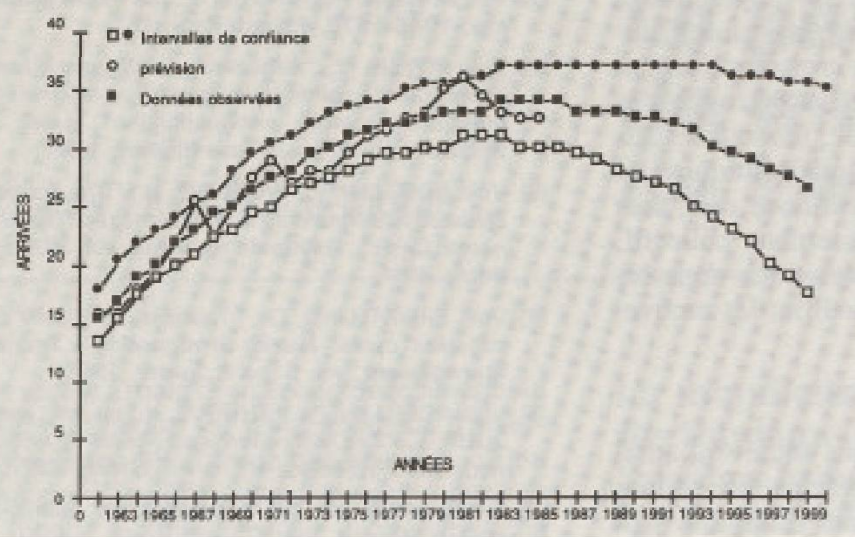

Graphique 2: Prévisions des arriveses des touristes en Amerique latine

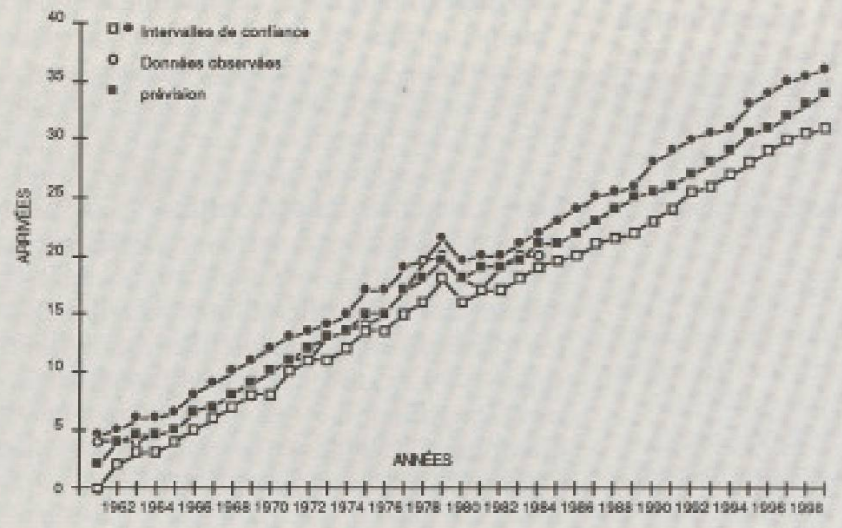

Graphique 3: Próvisions des arrivées des touristes en Europe

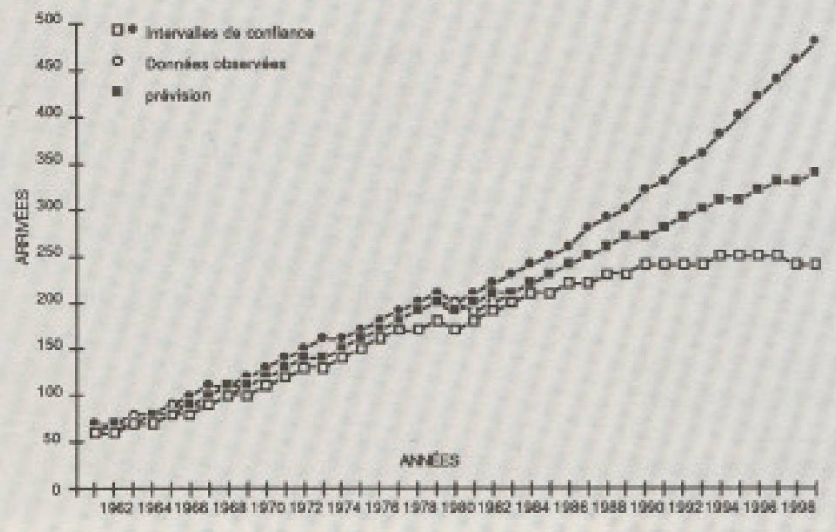

\section{Graphique 4: Prévisions des arrivées des touristes au Moyen-Orient}

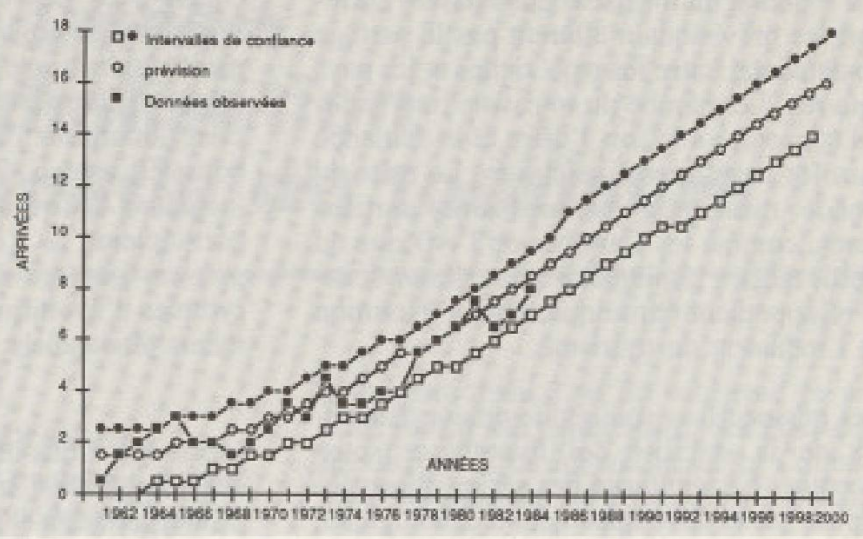

Graphique 5: Prévisions des arrivées des touristes en Afrique

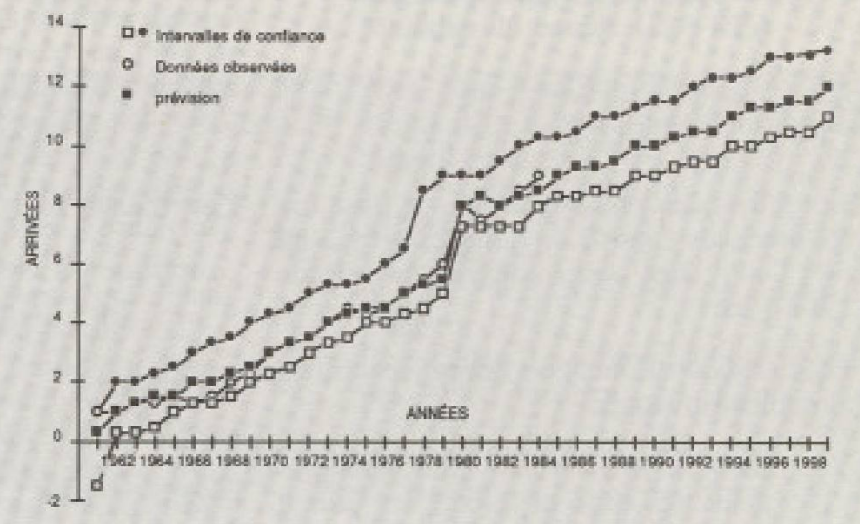

Graphique 6: Prévisions des arrivées du touristes dans rAsie de l'est. et le Pacilique

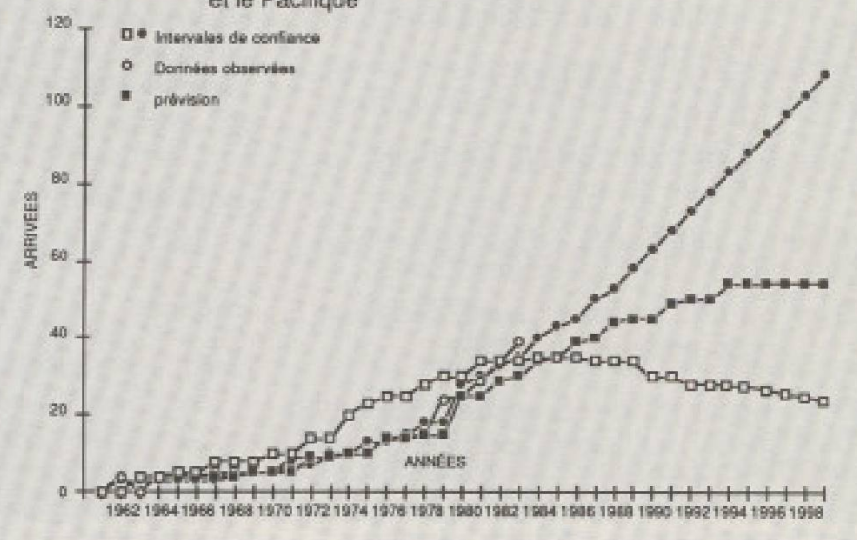

Graphique 7: Právisions des arrivées des touristes en Asie du sud

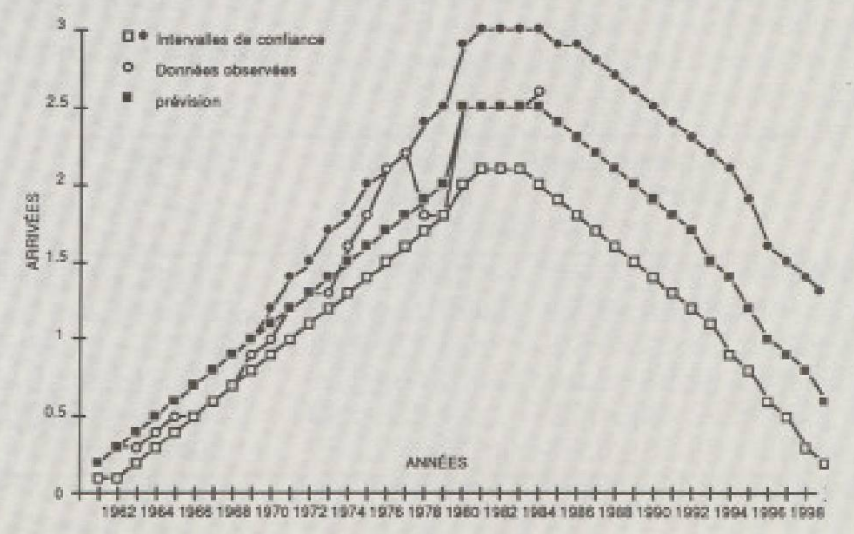


Les modifications dans la répartition du marche mondial du tourisme que laissent entrevoir les prévisions n'altèrent pas le partage fondamental entre les pays riches et les pays pauvres. Le marché du tourisme international concentre a la fois l'offre et la demande dans les pays à revenus élevés. Le reste du monde, mis à part quelques zones particulières, comme les Caraïbes ou l'A frique du Nord, est peu impliqué, à cause de son sousdéveloppement chronique, dans l'évolution de l'industrie du tourisme.

Ces perspectives sont évidemment tributaires de la stabilité des hypothèses sousjacentes. La régularité des séries observées repose sur le développement technologique, en particulier le développement des transports, sur la possibilité de mettre en rapport des localisations comparativement avanta- geuses (par leurs caractéristiques, par leurs coûts) avec des couches de population à revenu relativement élevé, sur l'enrichissement systématique lié à la croissance économique. L'impact de la crise pétrolière, la crise des années 1981-1982, les soubresauts boursiers actuels, les tensions politiques, les inégalités des régions du monde dans leur participation à l'enrichissement collectif, peuvent générer des fluctuations dans les rythmes d'évolution et modifier les mouvements tendanciels.

Les orientations discernces soulèvent aussi de nombreuses interrogations en termes d'interprétation et en termes d'impacts économiques, politiques et sociaux. Une analyse des données réelles et prévues s'imposerait dans cette perspective.
Notes at rifturences

11] Voir a ce sujat: FOURASTIE, J., Les trente glorieu ses: 1946-1975, Fayard, Paris, 1979.

(2) Voir: BOYER, M., "Evolution sociologique de tou risme. Continute du tourisme rare au towisme de masse et rupture contemporaine". Loisir ot Société, wol. III, no 1, Trois-Rivières, 1980.

(3) Voir: BOERJAN, P., VANHOVE, N., "The Towism Demand Reconsidered in the Context of the Econowic Crisis". Revue de tourisme, vol, 39, no 2 Berne, 1984.

(4) Voir: FRECHTLING, D., "Towism kends and the business cycle. Tourim in recossion", Tourism Management, wol, 3, no 4, Surroy, 1982.

(5) Voir; BOERJAN, P., VANHOVE, N., op. cit.; aussi JOLIN, L. "Lex tawis gubens au-dessus de FAthen tique Nord". Thores, vol. 6, no 1, mars 1987

(6) Etude pllote sur les prévisions du tourisme O.M.T., Madrid, 1981.

(7) GIACOBBI, M. et ROUX, J.P., Le dollar, Monnaie américaine ou monnaie mondiale?. Paris, 1986. chap. 4.

\section{ANNEXE I}

Les prévisions présentées dans le texte ont été obtenues à partir de données provenant de I'OMT. Les séries couvrent la période 1962-1985.

Les relations sélectionnées ont eu recours aux variables suivantes:

$\mathrm{Y}_{\text {in }}=$ nombre d'arrivées internationales annuelles dans la région $\mathrm{i}$ et durant l'année t

$\mathrm{m}=$ le monde

$\mathrm{i}=1$ désigne l'Amérique du Nord

$\mathrm{i}=2$ désigne l'Amérique Latine

$i=3$ l'Europe

$\mathrm{i}=4$ le Moyen-Orient

$\mathrm{i}=5 \mathrm{l}^{\prime}$ Afrique

$\mathrm{i}=6$ l'Asie de l'est; le Pacifique

$\mathrm{i}=7 \mathrm{l}^{\prime}$ Asie du sud

$\mathrm{T}_{1}=$ désigne le temps $1962,1963,1964, \ldots$

$\mathrm{D}_{\mathrm{t}}=$ est une variable muette désignant la crise postérieure à 1981 .

$\mathrm{D}=1$ pour les années postérieures à 1981

$\mathrm{D}=0$ pour les années antérieures à 1982
Les relations sélectionnées sont les suivantes:

Amérique du Nord:

$Y_{\text {if }}=a+b T_{1}+c D_{1}+U_{1}$

Europe:

$\operatorname{Ln}\left(Y_{3 s}\right)=a+b \operatorname{Ln}\left(T_{2}\right)+c D_{1}+U_{1}$

Moyen-Orient:

$\mathrm{Y}_{4 !}=\mathrm{a}+\mathrm{bLn}\left(\mathrm{T}_{7}\right)+c \mathrm{~T}^{2}+\mathrm{U}_{\mathrm{t}}$

Afrique:

$\mathrm{Y}_{51}=\mathrm{a}+\mathrm{b} \operatorname{Ln}\left(\mathrm{T}_{1}\right)+\mathrm{cT}_{\mathrm{t}}^{2}+\mathrm{U}_{\mathrm{s}}$

Asie de l'est et Pacifique:

$\operatorname{Ln}\left(\mathrm{Y}_{\mathrm{ba}}\right)=+\mathrm{a}+\mathrm{b} \operatorname{Ln}\left(\mathrm{T}_{3}\right)+\mathrm{CT}^{2}{ }_{1}+\mathrm{dD}_{1}+\mathrm{U}_{\mathrm{g}}$

Asie du Sud:

$\operatorname{Ln}\left(\mathrm{Y}_{7 \mathrm{t}}\right)=+\mathrm{a}+\mathrm{b} \mathrm{T}_{1}+\mathrm{CT}_{\mathrm{t}}^{2}+\mathrm{U}_{\mathrm{t}}$

Monde:

$\mathrm{Ymt}=\sum_{i=1}^{7} \quad$ Yit
Les estimations des paramètres ont été obtenues par moindres carrés ordinaires. $\mathrm{La}$ sélection des relations s'est préoccupée de minimiser l'autocorrélation résiduelle. Dans les relations retenues, le $\mathrm{R}^{2}$ est toujours supérieur à .95 et les coefficients statistiquement significatifs. Les prévisions ont été effectuées à l'aide du logiciel S.A.S. sur l'ordinateur Amdahl de I'UQAM.

\section{ANNEXE II}

Prévision du nombre d'arrivées internationales

Regions du monde

\begin{tabular}{lllllll}
\hline $\begin{array}{l}\text { Amérique du } \\
\text { Nord }\end{array}$ & $\begin{array}{l}\text { Amérique } \\
\text { latine }\end{array}$ & Europe & $\begin{array}{l}\text { Moyen- } \\
\text { Orient }\end{array}$ & Afrique & $\begin{array}{l}\text { Asie de } \\
\text { l'est; } \\
\text { Pacifique }\end{array}$ & $\begin{array}{l}\text { Asie du } \\
\text { Sud }\end{array}$ \\
\hline $198633.51 \pm 3.40$ & $21.23 \pm 1.81$ & $231.82 \pm 21.31$ & $7.67 \pm 1.28$ & $9.16 \pm 80$ & $35.39 \pm 7.04$ \\
$198733.38 \pm 3.59$ & $22.15 \pm 1.98$ & $240.51 \pm 24.14$ & $8.11 \pm 1.34$ & $9.31 \pm .88$ & $37.69 \pm 8.22$ & $2.40 \pm .59$ \\
$198833.20 \pm 3.79$ & $23.07 \pm 2.06$ & $249.15 \pm 27.73$ & $8.56 \pm 1.43$ & $9.49 \pm .92$ & $39.94 \pm 9.70$ & $2.19 \pm .69$ \\
$198932.95 \pm 4.04$ & $24.00 \pm 2.10$ & $257.71 \pm 32.10$ & $9.02 \pm 1.52$ & $2.70 \pm .93$ & $42.09 \pm 11.53$ & $2.07 \pm .74$ \\
1990 & $32.64 \pm 4.33$ & $24.93 \pm 2.14$ & $266.70 \pm 36.72$ & $9.50 \pm 1.63$ & $9.91 \pm .95$ & $44.14 \pm 13.68$ \\
$199530.20 \pm 6.25$ & $29.57 \pm 2.39$ & $306.12 \pm 75.32$ & $11.91 \pm 2.82$ & $10.98 \pm 1.03$ & $51.93 \pm 29.8$ & $1.18 \pm .83$ \\
\hline
\end{tabular}

